
and Surfaces A, 2014, 460:429-440. http://dx.doi.org/10.1016/j.colsurfa.2014.01.043

\title{
PEGylation of surfacted magnetite core-shell nanoparticles for biomedical application
}

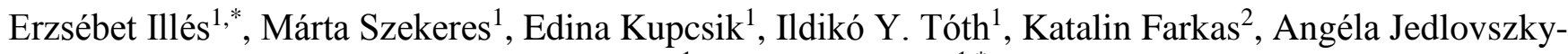 \\ Hajdú $^{1}$, Etelka Tombácz ${ }^{1, *}$
}

${ }^{1}$ Department of Physical Chemistry and Materials Science, University of Szeged, Aradi Vt. 1, H-6720 Szeged, Hungary

${ }^{2}$ Department of Laboratory Medicine, University of Szeged, Semmelweis u. 6, H-6720 Szeged, Hungary

Corresponding authors: E. Illés (illese@ chem.hu) and E. Tombácz (tombacz@ chem.u-szeged.hu) Elsevier ECIS2013 Best Poster Prize awarded contribution

Present address: A. Jedlovszky-Hajdú, Laboratory of Nanochemistry, Department of Biophysics and Radiation Biology, Semmelweis University, H-1089 Budapest, Nagyvárad tér 4, Hungary.

\section{Graphical abstract}
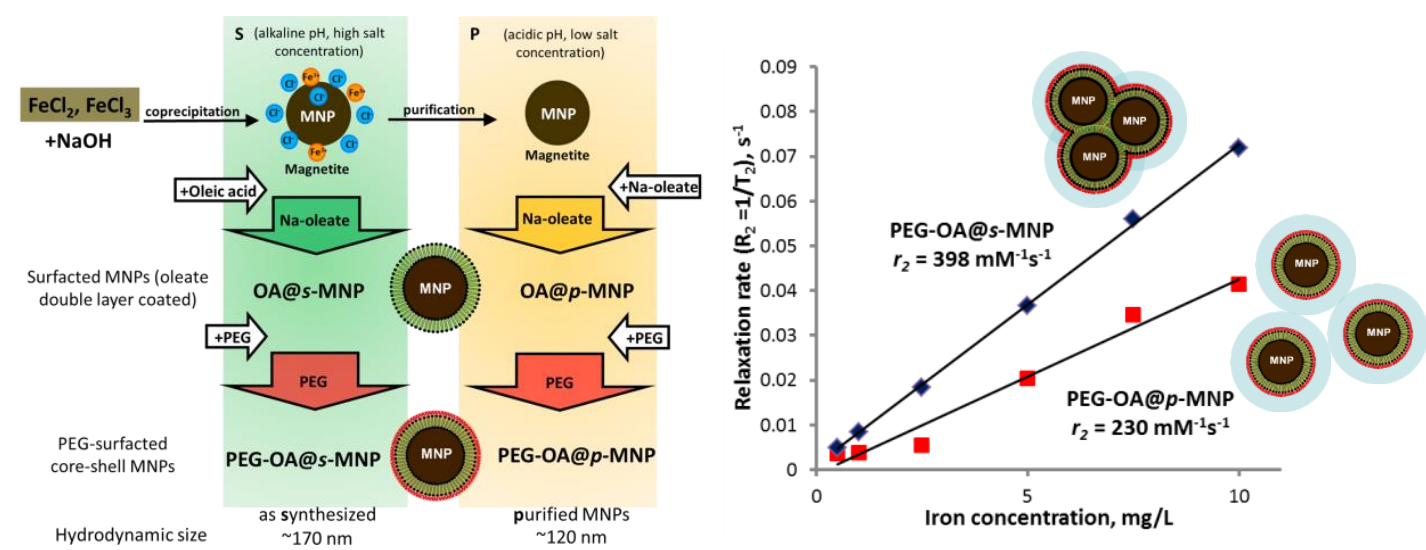

\section{Highlights}

- The mechanisms of oleate adsorption on as-synthesized and purified MNPs are different.

- PEG adsorption increases the weight of steric component in electrosteric stabilization by oleate.

- MNPs become clustered upon OA adsorption; cluster size is larger for as-synthesized MNPs.

- PEG-surfacted MNPs do not show cytotoxicity and hemolytic potential on human blood.

- MRI $T_{2}$ enhancement of as-synthesized MNPs supports higher degree of clustering.

\section{Abstract}

The surface of oleate double layer coated (surfacted) magnetite nanoparticles (OA@MNPs) was coated by PEG (poly(ethylene glycol) of $\mathrm{M}_{\mathrm{w}}=1000,4000$ or $20000 \mathrm{Da}$, respectively, to get core-shell structured nanomagnets. The oleate bilayers were prepared in two different ways; (i) oleic acid was added directly into the magnetite co-precipitation mixture containing the MNPs to obtain OA@ $s$-MNP samples $-s$-MNP standing for "as-synthesized MNP" and (ii) sodium oleate (oleate anion, OA) was added to the purified MNPs to obtain OA@p-MNP samples - $p$-MNP standing for "purified MNP". The effect of the surfactant addition method on the $\mathrm{pH}$ - and ionic strength-dependent stability (dynamic laser light scattering and laser-Doppler electrophoresis experiments), the biomedical applicability (MRI measurements) and the biocompatibility (blood sedimentation and blood smear tests) of the coreshell MNPs was studied. Different mechanisms of oleate adsorption were found in ATR FT-IR experiments (inner sphere surface complexation via ligand exchange for the $s$-MNPs and additional $\mathrm{H}$-bonding for the $p$-MNPs), suggesting different behaviour. The colloidal stability and salt tolerance of the two kinds of OA@MNPs were similar, but the hydrodynamic diameter of the OA@s-MNP was considerably larger than that of OA@p-MNP. In accordance with this, the $r_{2}$ relaxation was also higher for the $s$-MNP samples ( 400 and $\sim 200 \mathrm{mM}^{-1} \mathrm{~s}^{-1}$, respectively). The physico-chemical tests indicate that the OA-coated MNPs form clusters and the degree of clustering of OA@s-MNPs is significantly 
greater than that of OA@p-MPNs. PEGylation does not appear to affect colloidal stability and salt tolerance meaningfully. The adsorption of PEG was proved experimentally. We have found that the PEG top layer decreases the electrostatic contribution, nevertheless increases the steric contribution of the original electrosteric stabilization caused by the OA double layer. However, an increase in the molecular weight above $1000 \mathrm{Da}$ and the amount of added PEG above $5 \mathrm{mmol} / \mathrm{g}$ gradually reduces the salt tolerance of the samples. The results indicate strong potential for biomedical application and biocompatibility of the PEGylated MNPs.

Keywords: magnetite, oleate double layer, PEG coating, biocompatibility, MRI contrast agents

\section{Introduction}

Aqueous dispersions of iron oxide (magnetite, $\mathrm{Fe}_{3} \mathrm{O}_{4}$ and maghemite, $\gamma-\mathrm{Fe}_{2} \mathrm{O}_{3}$ ) nanoparticles (nanomagnets) are in the centre of recent studies due to their potential application in biomedicine (i.e., theranostics - MRI contrast agents, magnetic hyperthermia, drug-delivery vehicles), in bioseparation (cells, oligonucleotides) and numerous technical applications [1-6]. Nanomagnets (SPIONs, superparamagnetic iron oxide nanoparticles or MNPs, magnetite nanoparticles) have to be specialized for the individual applications; thus the design of customized MNPs and their colloidal stability have been under intense investigation [2-9].

Nanomagnets are suitable for magnetic fluid (MF) preparation, the magnetic nanoparticles of which resist aggregation even in the presence of strong magnetic field [2-9]. Aqueous magnetic fluids designed for biomedical application should be non-toxic, biocompatible, chemically stable, and sufficiently monodispersed [10,11]. Colloidal stability has crucial importance under physiological conditions, because particle aggregation in blood vessels can be disastrous. For biomedical applications, a protective layer is formed on the surface of the particles via specific interactions or chemical bonding between the active sites of iron oxides and the coating molecules. This coating of MNPs prevents the aggregation and can hinder chemical and biological degradation of the particles. Adsorption of small molecules, such as citric acid, fatty acids (especially the oleic acid), amino acids, various natural molecules (e.g., lecithin) and their combination have been applied to cover magnetic nanoparticles [1,11-22]. Biocompatible SPION coating can be also achieved by using macromolecules, e.g., homopolymers (PVA, PVP, PAA, PEG (polyethylene-glycol)) [10,16,18,19,23-28], copolymers (PEO-PPO(polypropylene oxide)-PEO (also known as Pluronics)) [4,29] or natural polymers (gelatine, polysaccharides, dextran) [11,21]. Even though the coating of nanomagnets with different surface modifiers leads to adequate stabilization, the blood plasma proteins can be adsorbed on their surfaces labelling them for opsonisation. In this case, the macrophages of the reticulo-endothelic system (RES) clear the nanoparticles from the blood and they cannot reach the target cells [4,29]. Protein adsorption can also deactivate the nanomagnets. Therefore, protection against the opsonisation is a key issue. The fundamental aim of research is to design biocompatible MNPs able to reach the target cells (without biological and/or chemical degradation in the presence of an external magnetic field) and exhibit the desired effect (e.g., contrasting, local heating or drug release) [2-9].

Magnetic Resonance Imaging (MRI) is one of the most widely used non-ionization imaging techniques, which provides high-resolution real-time anatomical information on the stage of diseases [2-6]. To enhance diagnostic efficiency, various contrast agents are applied. Complexes of gadolinium (e.g., Magnevist, Dotarem) are used actually in clinical practice due to their excellent superparamagnetic properties, i.e., the presence of 7 unpaired electrons. The main disadvantage of the application of gadolinium complexes is the formation of toxic by-products during the excretion [30-32]. Although dextran-coated superparamagnetic iron oxide nanoparticles have already been introduced in clinical trials as MRI contrast agents (e.g. Resovist, Endorem), the practical implementation of SPIONs is delayed [10,33]. The possibilities of preparation of newer contrast agents for specific interaction with different cells or tissues and their combination with drugs are still under intense research [2-9]. According to recently reported results $[5,10,11,20,23,26,28]$, no cytotoxicity and organ specific side-effect of SPIONs were observed up to now, which makes them a promising candidate for biomedical applications. 
PEGylation, i.e., coating of MNPs by PEG is one of the most favoured ways to ensure biocompatibility $[2-4,18,19,26,28]$. The strongly hydrated PEO-chains prevent the adsorption of blood proteins primarily due to steric repulsion [8,29]. Consequently, the hydrophilic coating increases the retention time of nanomagnets in the circulation system. According to literature sources, two main methods of PEGylation prevail [2,3,7,25]. The first is physico-chemical binding of PEG or functionalized PEG to the bare or previously modified (usually oleate covered) surface of magnetite. The other is the chemical coupling either in situ during the particle precipitation or chemisorption of functionalized PEG on the nanoparticle surface.

In this work we have prepared PEGylated nanomagnets by physico-chemical adsorption of poly(ethylene glycol) chains on the MNP surface previously coated by a sodium oleate double layer (surfacted MNP). We have studied the effect of the synthesis conditions on the colloidal stability, biomedical applicability and biocompatibility $[10,11]$ of the PEGylated nanomagnets. Two different strategies of oleate addition during the synthesis are compared: (i) Oleic acid was added to the as-prepared MNPs introduced directly into the alkaline synthesis medium where oleate anions (OA) form in situ, which is a broadly utilized strategy of magnetic fluid synthesis. (ii) Sodium oleate was added to the carefully purified MNPs at pH=3-4.

As surface modified MNPs can be prepared in several different ways [14,19,22], it has a great importance to compare the physico-chemical properties, bio-applicability and biocompatibility of the products prepared according to different methodologies. We have chosen two radically different approaches for the aims of comparison: "one-pot" synthesis with addition of reactants into the reaction mixture of MNP preparation and the more complicated synthesis process with separating and purifying steps and finally coating the MNPs.

\section{Materials and methods}

\subsection{Materials}

The $\mathrm{FeCl}_{2}$ and $\mathrm{FeCl}_{3}$ salts for magnetite synthesis were analytical grade reagents obtained from Molar, Hungary. The mean diameter of the resulting MNPs determined by transmission electron microscopy was $\sim 10$ nm [11]. The synthesized compound was identified as magnetite based on the X-ray diffraction pattern (JCPDS database [1]), characteristic black colour and strong magnetism. Oleic acid, sodium oleate (oleate anion, OA) and poly(ethylene glycol) (PEG1000, $\mathrm{M}_{\mathrm{W}}=1000 ;$ PEG4000, $\mathrm{M}_{\mathrm{w}}=4000$ and PEG20000, $\mathrm{M}_{\mathrm{w}}=20000 \mathrm{Da}$ ) were purchased from Sigma Aldrich. $\mathrm{NaCl}, \mathrm{HCl}$ and $\mathrm{NaOH}$, analytical grade products of Molar (Hungary) were used to set the $\mathrm{pH}$ and ionic strength. Milli-Q water was used.

\subsection{Synthesis of PEG-surfacted magnetite core-shell nanomagnets}

The MNP synthesis was performed according to the method of alkaline hydrolysis of $\mathrm{Fe}(\mathrm{II})$ and $\mathrm{Fe}(\mathrm{III})$ salts, detailed in our previous publication [35]. Since under atmospheric conditions the presence of oxygen enhances the oxidation processes, $10 \%$ excess of $\mathrm{Fe}(\mathrm{II})$ salt was used during the synthesis in order to maintain the stoichiometric $\mathrm{Fe}^{3+} / \mathrm{Fe}^{2+}$ ratio for magnetite $\left(\mathrm{Fe}_{3} \mathrm{O}_{4}\right)$ formation. The applied purification procedure involved magnetic separation as well [12], to reach less polydisperse iron oxide samples. The coating layer was prepared in two steps; first, the oleate covered (surfacted) magnetite nanoparticles were synthesized, then PEG molecules were attached to the surfacted nanomagnets. Two different synthesis procedures were applied, schematically presented in Fig. 1. In the first procedure, a calculated amount of oleic acid ( $2 \mathrm{mmol} / \mathrm{g}$ with respect to the iron oxide content) was added to the MNP synthesis mixture right after the precipitation of magnetite nanoparticles according to the previously published method [12,34]. The $\mathrm{pH}$ of the synthesis mixture was $\sim 11.3$ and it had a high salt concentration (the specific conductivity was $\sim 90 \mathrm{mS} / \mathrm{cm}$ ). Since the $\mathrm{pH}$ of the medium is highly basic, oleic acid dissociates instantaneously by in-situ formation of oleate anions (OA) that bind to the MNP surface. The first OA layer makes the surface hydrophobic. 


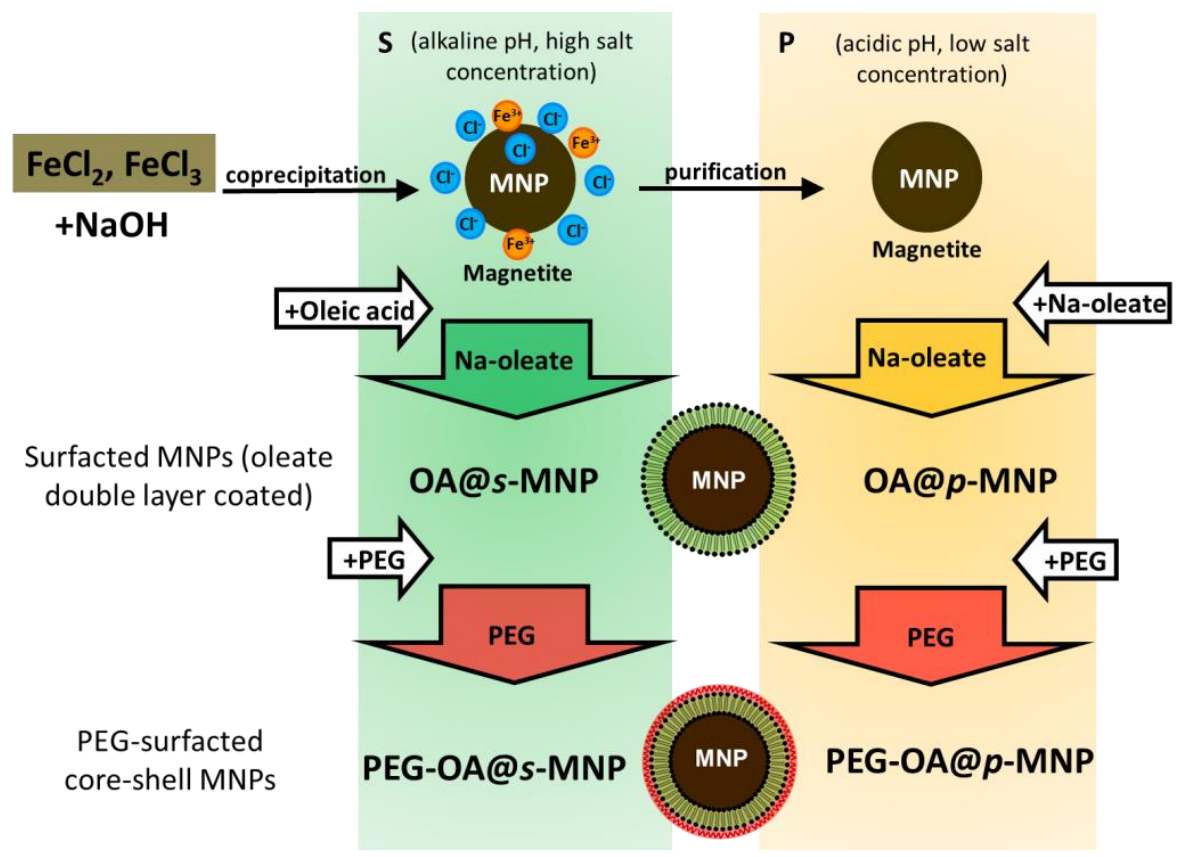

Fig. 1. Schematic presentation of the two synthesis routes of PEG-surfacted MNPs. OA@ $s$-MNP preparation route denoted by $S$ (left) - addition of oleic acid to the strongly alkaline synthesis mixture right after the co-precipitation of magnetite nanoparticles and in-situ formation of sodium oleate (OA); and OA@p-MNP preparation route denoted by $\mathrm{P}$ (right) - addition of OA to the purified magnetite nanoparticles at $\mathrm{pH}$ 3-4. Various amounts of PEG (5- $120 \mathrm{mmol}$ EO/g MNP) with molecular weight of 1000, 4000 and $20000 \mathrm{Da}$, respectively, were added to the surfacted nanomagnets and PEG top shells are formed (PEGOA@s-MNP and PEG-OA@p-MNPs).

The second OA layer becomes adsorbed via hydrophobic interaction and makes the surface hydrophilic again. The outer surface of the coated MNPs becomes negatively charged due to the dissociated carboxylate groups of the oleate coating. The surfacted nanomagnets obtained by this method are designated as OA@sMNP, because OA formed in-situ from the added oleic acid are adsorbed on the surface of as-synthesized MNPs (s-MNPs). In the second procedure, the magnetite nanoparticles were separated from the synthesis mixture and purified. Magnetic separation and washing with distilled water were repeated several times. Finally, the dispersions were dialysed against $1 \mathrm{mM} \mathrm{HCl}$ solution to eliminate excess salt. The average particle diameter of the purified MNPs was $\sim 10 \mathrm{~nm}$, the $\mathrm{pH}$ was $\sim 3.6$ and the conductivity was measured as 295 $\mu \mathrm{S} / \mathrm{cm}$. Sodium oleate was added from aqueous solution to the acidic dispersion of purified MNPs in the same amount as oleic acid in the first strategy $(2 \mathrm{mmol} / \mathrm{g})$. The MNP surface is positively charged at this $\mathrm{pH}$; thus, the adsorption of oleate anions $(\mathrm{OA})$ is promoted by electrostatic attraction. However, at low $\mathrm{pH}$ the adsorption of non-dissociated oleic acid is also probable. The obtained samples are designated as OA@p-MNP, $p$-MNP meaning purified magnetite nanoparticles. The PEG top shell on both types of surfacted nanomagnets was prepared in the same way. Aqueous solutions of PEG1000, PEG4000 and PEG2000, respectively, were added to reach final loadings between 5 and $120 \mathrm{mmol} / \mathrm{g}$. Throughout the paper, the concentration of PEG is given in mmoles of EO units per grams of MNP.

\subsection{Infrared spectroscopy (ATR FT-IR)}

ATR FT-IR spectra were recorded with a Bio-Rad Digilab Division FTS-65A/896 spectrometer (with DTGS detector), using a Harrick's Meridian Split Pea Diamond ATR accessory. The absorbance of the samples was measured in single reflection mode over the 400-4000 $\mathrm{cm}^{-1}$ range (with resolution of $2 \mathrm{~cm}^{-1}$ ), accumulating 256 scans. Magnetite dispersions (MNP, OA@s-MNP, OA@p-MNP and PEG-OA@p-MNP), aqueous OA (sodium oleate) and PEG solutions, and liquid oleic acid were cast and dried on the crystal surface. For FTIR spectra, PEG20000 and PEG20000-coated MNPs were used. Except for oleic acid, the pH 
of all samples was set to $~ 6.5$ and the ionic strength was $10 \mathrm{mM}$. The PEG loading of the PEG-OA@p-MNP sample was $120 \mathrm{mmol} / \mathrm{g}$. The background spectra were measured on clean and dry diamond crystal.

\subsection{Electrophoresis measurements}

Electrophoretic mobilities of the uncoated magnetite, the surfacted nanomagnets (OA@s-MNP and OA@p-MNP) and the PEG-surfacted particles (PEG-OA@ $s$-MNP and PEG-OA@ $p$-MNP) were measured at $25 \pm 0.1{ }^{\circ} \mathrm{C}$ in a Nano ZS (Malvern) dynamic light scattering (DLS) apparatus with a $4 \mathrm{~mW} \mathrm{He}-\mathrm{Ne}$ laser source $(\lambda=633 \mathrm{~nm}$ ), using disposable zeta cells (DTS 1061). The accuracy of the measurements is $\pm 5 \mathrm{mV}$ and the zeta-standard of Malvern $(-55 \pm 5 \mathrm{mV})$ was used for calibration. The dispersions were diluted to give an optimal intensity of $\sim 10^{5}$ counts per second. Prior to the measurements, the samples were homogenized in an ultrasonic bath for $10 \mathrm{~s}$, after which 2 min relaxation was allowed. The influence of the added amount of PEG20000 (5, 15, 50 and $120 \mathrm{mmol} / \mathrm{g})$ on the electrokinetic potential of the OA@ MNPs was determined at $\mathrm{pH} \sim 6.5$ and $\mathrm{I}=10 \mathrm{mM}(\mathrm{NaCl})$. The effect of $\mathrm{pH}$ on the electrophoretic mobilities of the naked and coated nanomagnets was studied from $\mathrm{pH} 2$ to $\sim 11$ at $\mathrm{I}=10 \mathrm{mM}$. The Smoluchowski equation was applied to convert electrophoretic mobilities to electrokinetic potential values.

\subsection{Dynamic light scattering (DLS)}

The average particle size of bare magnetite, the oleate covered and the PEG-surfacted core-shell nanoparticles were determined at $25 \pm 0.1{ }^{\circ} \mathrm{C}$ using a Nano ZS apparatus (Malvern) operating in backscattering mode at an angle of $173^{\circ}$. The solution conditions were the same as in the electrophoresis measurements: the added amounts of PEG20000 were 5, 15, 50 and $120 \mathrm{mmol} / \mathrm{g}$, the $\mathrm{pH}$ range was $\sim 2$ to $\sim 11$ and the ionic strength $\mathrm{I}=10 \mathrm{mM}$. The aggregation state of the bare or the coated nanoparticles in the aqueous dispersions was characterized by the changes in the intensity average values (Z-Ave) of the hydrodynamic diameter. We used the second- or third-order cumulant fit of the autocorrelation functions, depending on the degree of polydispersity.

\subsection{Salt tolerance tests}

Coagulation kinetic measurements were performed to determine the stabilization effect of the PEG1000, PEG4000 and PEG20000 top shells of surfacted magnetite nanoparticles. The PEG loadings were $5,15,50$ and $120 \mathrm{mmol} / \mathrm{g}$. The size evolution in time was followed by dynamic light scattering using a NanoZS instrument (Malvern). The change in the hydrodynamic diameter (Z-Ave) was measured at $25 \pm 0.1{ }^{\circ} \mathrm{C}$ for 10 min with a resolution of $60 \mathrm{~s}$. The slope of the kinetic curves $\mathrm{dZ}$-Ave/dt $=\mathrm{f}(\mathrm{t})$ is proportional to the coagulation rate [36,37]. Coagulation was induced by increasing the concentration of $\mathrm{NaCl}$ in the dispersion medium at $\mathrm{pH}$ 6.5. The stability ratio (W) was calculated as the ratio of the initial slopes of the kinetic curves at fast and slow coagulation. The salt tolerance of the samples is given as the critical coagulation concentration (CCC) obtained from the stability ratio $(\log W)$ vs. electrolyte concentration $(\log \mathrm{c})$ functions $[10,35,38]$.

\subsection{Magnetic Resonance Imaging (MRI)}

The MRI contrast enhancement efficiency of the pegilated nanomagnets was studied by determining the $\mathrm{T}_{1}$ (longitudinal) and $\mathrm{T}_{2}$ (transversal) relaxation times and calculating $\mathrm{r}_{1}$ and $\mathrm{r}_{2}$ relaxivities at a field strength of $1.5 \mathrm{~T}$ at $20{ }^{\circ} \mathrm{C}$ by using a clinical MRI instrument GE Excite HDxt (GE Medical Systems, Milwaukee, WI). A standard "birdcage" head coil was applied in the experiments.

The OA@s-MNP, OA@p-MNP PEG-OA@s-MNP and PEG-OA@s-MNP samples contained 2 $\mathrm{mmol} / \mathrm{g}$ of oleate. The amount of PEG20000 was $120 \mathrm{mmol} / \mathrm{g}$. The final concentrations of iron $(\mathrm{Fe})$ in the examined MNP dispersions were 0.5, 1, 2.5, 5, 7.5 and $10 \mathrm{mg} / \mathrm{L}$. The $\mathrm{pH}$ of the samples was $\sim 6.5$ and the ionic strength $0.01 \mathrm{M}$. The average hydrodynamic diameter of the particles was checked by DLS prior to the MRI measurements. 
$20 \mathrm{~mL}$ of samples was placed in a plastic box filled with water and put in the centre of the head coil. The $T_{1}$ relaxation values were determined applying the inversion recovery spin echo imaging sequence [39] with inversion times (TI) of 50, 100, 200, 400, 800, 1400, 2200 and $3600 \mathrm{~ms}$. The $\mathrm{T}_{2}$ relaxation times were estimated applying the conventional spin echo imaging sequence [39] with echo times (TE) of 10, 20, 30, 40, 60, 120, 180 and $240 \mathrm{~ms}$. Other imaging parameters were kept constant.

The $T_{1}$ and $T_{2}$ relaxation values were determined from mean signal intensities within a manually drawn region of interest (ROI) for each sample. The signal intensity vs. TI or TE functions were exponentially fitted using Origin Pro 8 software (OriginLab Corporation, Northampton, MA) based on the following equations:

$$
\begin{array}{ll}
y=\left|A+B \times \exp \left(-T I / T_{1}\right)\right| & \text { for T1 relaxation } \\
y=A \times \exp \left(-T E / T_{2}\right) & \text { for T2 relaxation, }
\end{array}
$$

where $\mathrm{y}$ is the signal intensity, and $\mathrm{A}$ and $\mathrm{B}$ are constants.

The values of $r_{1}$ and $r_{2}$ relaxivities were calculated as the slopes of the $1 / T_{1}$ and $1 / T_{2}$ vs. Fe concentration plots [40]. The goodness of fitting was estimated by the coefficient of determination (R-squared).

\subsection{Biocompatibility tests}

Blood biocompatibility of PEG-OA@p-MNP, i.e., its influence on the colloidal state of whole blood of three healthy volunteers (Donors \#1 to \#3) was studied by erythrocyte sedimentation rate (ESR) experiments, using a Sedi-15 automated blood sedimentation instrument and Seditainer 1.8 Vacutainer tubes (BD Inc., USA). The PEG-OA@p-MNP sample $(\mathrm{pH} \sim 6.5, \mathrm{I}=0.01 \mathrm{M})$ prepared for the ESR measurements contained $2 \mathrm{mmol} / \mathrm{g}$ of oleate and $5 \mathrm{mmol} / \mathrm{g}$ of PEG20000 and mixed with the blood at a 1:5 volumetric ratio. The final concentration of MNP in the citrate-anticoagulated tubes was $0.16 \mathrm{mg} / \mathrm{L}$ [11]. The accuracy of the ESR measurements is $\pm 3 \mathrm{~mm} / \mathrm{h}$ as given in the manual of Sedi- 15 .

Blood smear experiments were performed using an automated slide preparation system Sysmex SP4000i (Biolyon, Dardilly, France) using the May-Grünwald Giemsa (MGG) staining technique. A CellaVisionTM DM96 (Cellavision AB, Ideon, Science Park, Lund, Sweden) instrument consisting of a slide feeder unit, a microscope, a camera and a computer was used for data acquisition and blood cell classification. The influence of the PEG-OA@p-MNP on the aggregation state of whole blood was tested at a volumetric ratio of 1:5 of nanomagnet dispersion to EDTA-anticoagulated blood sample. The samples were mixed and allowed to stand at $25^{\circ} \mathrm{C}$ for 10 minutes. Prior to preparing of smear the mixtures were homogenized again.

\section{Results and discussion}

\subsection{OA and PEG adsorption}

The formation of oleate layers on MNPs and PEG20000 top shell on the surfacted nanomagnets were studied by infrared spectroscopy. The results are shown in Figs. 2 and 3. The spectrum of the bare iron oxide nanoparticles was substracted from that of the variously coated nanomagnets, i.e. OA@MNPs and PEGOA@p-MNP. The FT-IR spectrum of sodium oleate (OA) dried on the diamond crystal (IRE) from aqueous solution at $\mathrm{pH} \sim 6.5$ (Fig. 2) shows three characteristic vibrations of the carboxylic groups: $1710 \mathrm{~cm}^{-1}$ (the $\mathrm{C}=\mathrm{O}$ band of the nondissociated caboxlys), $1558 \mathrm{~cm}^{-1}$ (the asymmetric $\mathrm{COO}^{-}$vibration) and $1406 \mathrm{~cm}^{-1}$ (the symmetric $\mathrm{COO}^{-}$vibration of the dissociated carboxylates) [41,42]. Another well expressed peak at $1462 \mathrm{~cm}^{-}$

1 is due to the scissoring bending mode of $\mathrm{CH}_{2}[43,44]$. The latter is one of the a major identification bands of saturated hydrocarbons found in general near $1460 \mathrm{~cm}^{-1}$ [45]. The $\mathrm{COO}^{-}$symmetric stretching mode (1406 $\mathrm{cm}^{-1}$ ) is strongly coupled with the different vibration modes of the aliphatic chain [44] and thus, it is not well resolved. In addition, the wide peak appearing at $1100 \mathrm{~cm}^{-1}$ has been assigned by J. Nájera [44] to the interaction of sodium oleate with water, which was not found in dry samples in his studies. For comparison, we present the FT-IR spectrum of pure oleic acid in Fig. 2. 


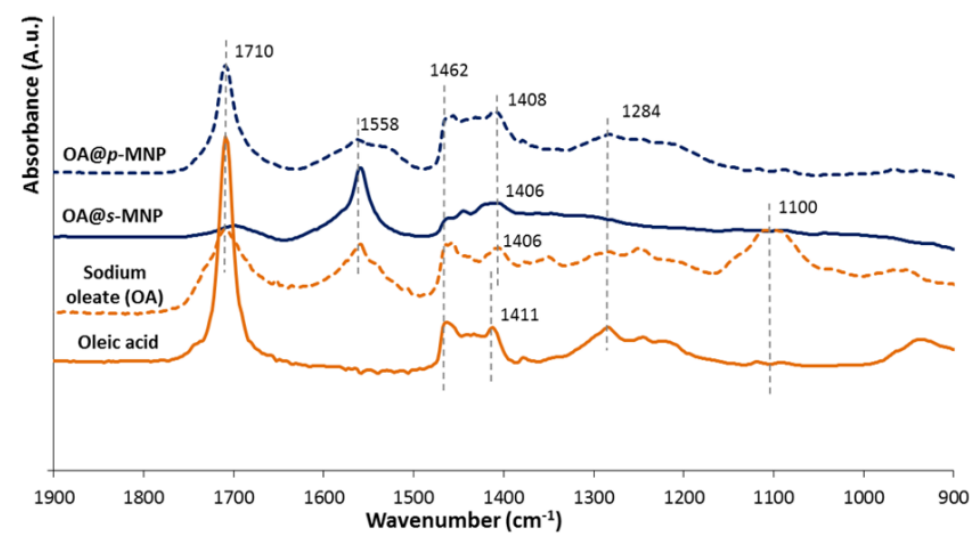

Fig. 2. The characteristic range $\left(900-1900 \mathrm{~cm}^{-1}\right)$ of the ATR FT-IR spectra of surfacted magnetite nanoparticles (OA@p-MNP and OA@s-MNP) together with the spectra of pure sodium oleate $(\mathrm{OA})$ and oleic acid. The samples were dried on the ATR crystal from $\mathrm{pH} \sim 6.5, \mathrm{I}=10 \mathrm{mM}$ medium (except for oleic acid).

The characteristic oleic acid bands, the $\mathrm{C}=\mathrm{O}$ streching at $1710 \mathrm{~cm}^{-1}$, the $\mathrm{C}-\mathrm{O}$ stretching at $1284 \mathrm{~cm}^{-1}$ and the $\mathrm{CH}_{2}$ bending at $1462 \mathrm{~cm}^{-1}$ fully coincide with literature data [45]. It is evident that in the absence of water the $1100 \mathrm{~cm}^{-1}$ peak found in the OA sample assigned to interaction with water [44] is also absent. The band observed at $1411 \mathrm{~cm}^{-1}$ can be assigned to $\mathrm{CH}_{2}$ and/or $\mathrm{CH}$ vibration modes of the aliphatic chain and it differs definitely from that in the OA sample (broadened by the symmetric stretching band of $\mathrm{COO}^{-}$and shifted to $\left.1406 \mathrm{~cm}^{-1}\right)$.

When oleic acid is added to the very alkaline suspension of MNPs for preparation of OA@s-MNP, the in situ formed sodium oleate interacts with the as-synthesized MNPs. The characteristic carbonyl stretching vibration at $1710 \mathrm{~cm}^{-1}$ disappears from the spectrum almost entirely; but the asymmetric and symmetric C$\mathrm{OO}^{-}$vibrations become well expressed at 1558 and $1406 \mathrm{~cm}^{-1}$. The latter bands clearly do not shift in the course of OA adsorption indicating that the environment of the carboxylate groups remains unchanged. The distance between the asymmetric and symmetric $\mathrm{COO}^{-}$peaks $\left(\Delta v_{\text {asym-sym }}\right)$ is $152 \mathrm{~cm}^{-1}$, which reveals bidentate coordination [41]. Bidentate chelate complexes can be formed with the surface Fe atoms, in which the state of OA is similar to that in the sodium salt form. The latter explains the absence of shifts in the FT-IR spectra. This process can occur via ligand exchange with the uncharged surface sites of MNP:

$$
\equiv \mathrm{Fe}-\mathrm{OH}+\mathrm{R}-\mathrm{COO}^{-} \rightarrow \quad \equiv \mathrm{Fe}-\mathrm{OOC}-\mathrm{R}+\mathrm{OH}^{-} .
$$

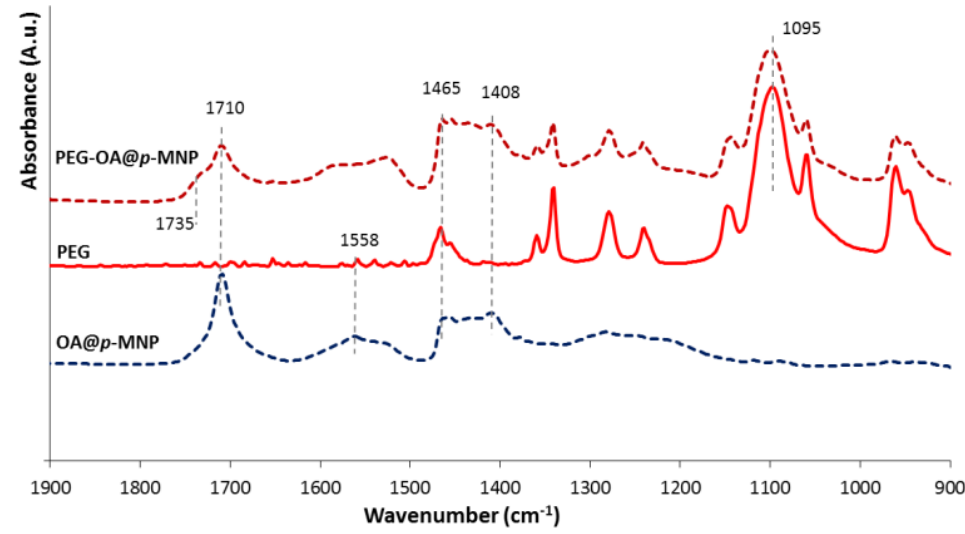

Fig. 3 The characteristic range $\left(900-1900 \mathrm{~cm}^{-1}\right)$ of the ATR FT-IR spectra of PEG20000-surfacted magnetite nanoparticles PEG-OA@ @-MNP pure PEG20000. The PEG loading is $120 \mathrm{mmol} \mathrm{EO/g}$. For reference, the spectrum of OA@p-MNP is added. 
As the $\mathrm{pH}$ of the dispersion of as-synthetized MNP (s-MNP) is high, the oleate anions prefer to adsorb only at the uncharged surface sites but not at the negatively charged $\equiv \mathrm{Fe}-\mathrm{O}^{-}$groups.

When sodium oleate is added to the purified MNPs (OA@p-MNP), a high-intensity band appears 1710 $\mathrm{cm}^{-1}$ (Fig. 2.) demonstrating the formation of a H-bonded structure with the positively charged surface sites of $\mathrm{MNP}\left(\mathrm{pH}_{\mathrm{pzc}} \sim 8\right)$ :

$\equiv \mathrm{Fe}-\mathrm{OH}_{2}{ }^{+}+\mathrm{R}-\mathrm{COO}^{-} \rightarrow \equiv \mathrm{Fe}-\mathrm{OH} . . \mathrm{HOOC}-\mathrm{R}$.

At the low $\mathrm{pH}$ of the dispersion medium of purified MNPs, undissociated oleic acid can be adsorbed to the neutral MNP surface sites forming H-bonds:

$$
\equiv \mathrm{Fe}-\mathrm{OH}+\mathrm{R}-\mathrm{COOH} \rightarrow \equiv \mathrm{Fe}-\mathrm{OH} \ldots \mathrm{HOOC}-\mathrm{R} .
$$

In addition, ligand exchange reaction is also possible with the uncharged surface sites of the processed MNP:

$$
\equiv \mathrm{Fe}-\mathrm{OH}+\mathrm{R}-\mathrm{COO}^{-} \rightarrow \equiv \mathrm{Fe}-\mathrm{OOC}-\mathrm{R}+\mathrm{OH}^{-}
$$

The latter explains the presence of the characteristic $\mathrm{COO}^{-}$bands (asymmetric at $1558 \mathrm{~cm}^{-1}$ and symmetric at $1408 \mathrm{~cm}^{-1}$ ) in the spectrum of OA@p-MNP, not shifted with regard to that of sodium oleate and OA@s-MNP. In addition, the sodium oleate anions are present in both s- and p- types of OA@MNP as the second adsorbed layer and contribute to the $\mathrm{COO}^{-}$vibrations. The relative intensities of the $\mathrm{C}=\mathrm{O}$ and the asymmetric $\mathrm{COO}^{-}$vibrations express the relative weight of the $\mathrm{H}$-bonded and bidentate chelate bonded states of $\mathrm{OA}$ in the adsorbed state.

The FT-IR spectrum of the PEG-OA@p-MNP sample in Fig. 3 shows, in principle, all the characteristic IR vibrations of both OA@p-MNP and PEG. Both the H-bonded $\left(1710 \mathrm{~cm}^{-1}\right)$ and bidentate chelated (the 1558 and $1408 \mathrm{~cm}^{-1}$ ) states [41] of adsorbed OA are observable and in addition, the characteristic absorption bands of PEG (specifically, the $\mathrm{CH}_{2}$ bending at $1465 \mathrm{~cm}^{-1}$ and the out-of plane $-\mathrm{O}-\mathrm{C}-\mathrm{C}-\mathrm{O}-$ stretching at $1095 \mathrm{~cm}^{-1}$ ) are superposed in the spectrum. The $1558 \mathrm{~cm}^{-1}$ vibration becomes buried in a wide absorption range of newly appearing peaks between 1587 and $1525 \mathrm{~cm}^{-1}$. These changes in the carboxylate stretching region can be due to the changes in the environment of the outer $-\mathrm{COO}^{-}$groups of the OA double layer because of PEG adsorption. In addition, a new absorption band appears in the spectrum of PEG-OA@pMNP centered at $\sim 1735 \mathrm{~cm}^{-1}$ as a shoulder missing from that of PEG and OA@p-MNP. This band is most likely due to the formation of ester linkages between the carboxyl groups of outer OA molecules and the terminal $\mathrm{OH}$ groups of PEG. It suggests that increasing the molecular weight reduces the number of connection possibilities of PEG with the oleate covered surface.

\subsection{Electrophoretic mobility of the core-shell MNPs}

The effect of surfactant and PEG adsorption on the electrokinetic potential of magnetite nanoparticles were studied at $\mathrm{pH}$ between $\sim 2$ and $\sim 11$. The amount of surfactant was that necessary to reach the double layer coating and PEG20000 loading were 5, 15, 50 and $120 \mathrm{mmol} / \mathrm{g}$. The results are shown in Fig. 4 (a) and (b).

The pH-dependent surface charge of naked magnetite (black symbols in Fig. 4a) displays a distinct IEP (isoelectric point) at $\mathrm{pH} \sim 8[14,15,35]$. When the bare purified nanomagnets become coated with an oleate double layer (red symbols), the $\mathrm{pH}$-dependence of the electrokinetic potential resembles that of carboxylic acids due to the carboxylic groups linked to the outer surface of MNPs. The IEP disappears. Owing to the gradual decrease in dissociation of the carboxylic groups of OA, the zeta potential approaches zero with decreasing $\mathrm{pH}$. Similar trend of the change in the electrokinetic potential of the nanoparticles with $\mathrm{pH}$ was observed for both as-synthesized and the purified MNP samples.

The shape of the $\mathrm{pH}$-dependent zeta potential curves is similar also for each PEG loading 5, 15, 50 and $120 \mathrm{mmol} / \mathrm{g}$. An increase in the added amount of PEG, however, shifts characteristically the electrokinetic 
potential values towards zero. Masoudi et al [28] observed the same type of shielding effect of the adsorbed PEG on zeta potentials. The decrease in the absolute values of zeta potentials due to PEG addition proves the accumulation of PEG at the carboxylated surface of OA@MNPs as it is sketched in Fig. 1.
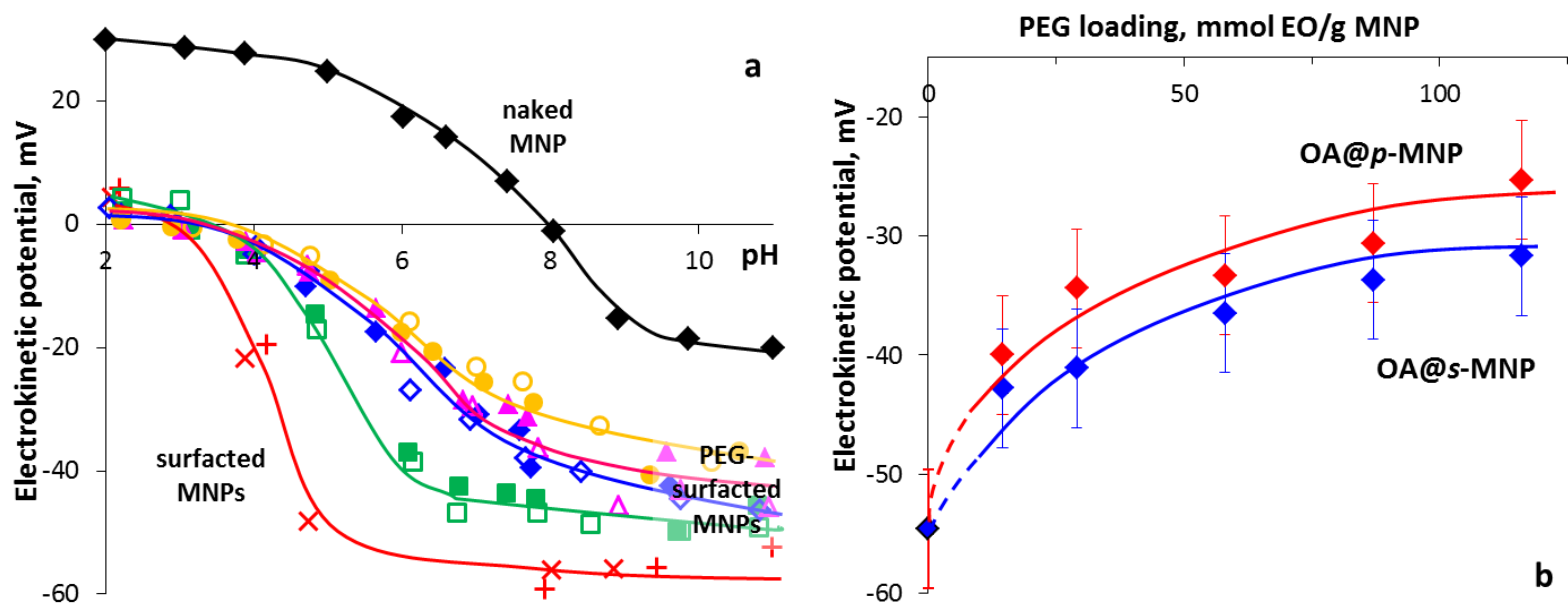

Fig. 4. (a) pH-dependent electrokinetic potential of naked (black), surfacted (red) and PEG-surfacted MNPs (5 (green square), 15 (blue diamond), 50 (magenta triangle) and 120 (orange circle) mmol EO/g of PEG2000) in the presence of $10 \mathrm{mM} \mathrm{NaCl}$. The open symbols represent the $s$-MNP and the full symbols the $p$-MNP samples. The accuracy of the measured potential values is $\pm 5 \mathrm{mV}$. (Lines are drawn as a guide for the eye.) (b) The change in the electrokinetic potential of the PEG-OA@p-MNP and PEG-OA@ $s$-MNP samples with PEG20000 loadings from 0 to $120 \mathrm{mmol} \mathrm{EO/g}$ at pH 6.5 in the presence of $10 \mathrm{mM} \mathrm{NaCl}$. (Lines are drawn as a guide for the eye.)

The adsorption of PEG on the surfacted MNPs at $\mathrm{pH} \sim 6.5$ leaded to systematically lower absolute values of electrokinetic potential for the as synthesized than that for the purified MNPs, as it is seen in Fig. $4 \mathrm{~b}$. This result suggests that under identical conditions the adsorption of PEG on the OA@s-MNPs can be somewhat larger than on OA@p-MNPs. However, the difference in the electrokinetic potentials is not significant enough to draw unambiguous conclusions regarding the probable difference in the adsorbed amounts.

\section{3. $p H$-dependent stability of the core-shell MNPs}

The results of dynamic light scattering experiments in Figs. 5a and b clearly show that the average hydrodynamic diameter (Z-Ave) of the PEG20000-surfacted core-shell MNPs increases abruptly at pH below $\sim 6$ for all PEG loadings $0,5,15,50$ and $120 \mathrm{mmol} / \mathrm{g}$. The approximately four-fold increase in the hydrodynamic diameter reveals particle aggregation, which is also supported by the increase in the polydispersity index (PDI) from about $0.15 \pm 0.02$ (Table 1) at $\mathrm{pH} \sim 6.5$ to above 0.4 at lower $\mathrm{pHs}$. The sizes of the colloidally stable, i.e., non-aggregated MNPs at $\mathrm{pH}>6$ are $170 \mathrm{~nm}$ for the OA@ $s$-MNPs and PEG-OA@sMNPs, and 120 nm for OA@p-MNPs and PEG-OA@p-MNPs. Both are considerably higher than the size of uncoated MNPs (Z-Ave $\sim 98 \mathrm{~nm}$, PDI=0.147 as shown in Table 1.).

The larger size of the colloidally stable PEG-surfacted MNPs reveals that these are possibly formed by a loose aggregation of the original MNPs. Their stability and approximately monodisperse size distribution allows to identify them as nanoparticle clusters. This observation is in harmony with SANS results of Adveev et al [16], revealing a clustered structure of surfacted and PEG-surfacted MNPs. As the Z-Ave values do not change with PEG addition at increased amounts, PEG does not seem to enhance the stability of the aggregates formed upon addition of OA or oleic acid. Because of the significantly larger hydrodynamic diameter of the as-synthesized MNPs we can conclude that they undergo significantly stronger clustering than the purified MNPs. 

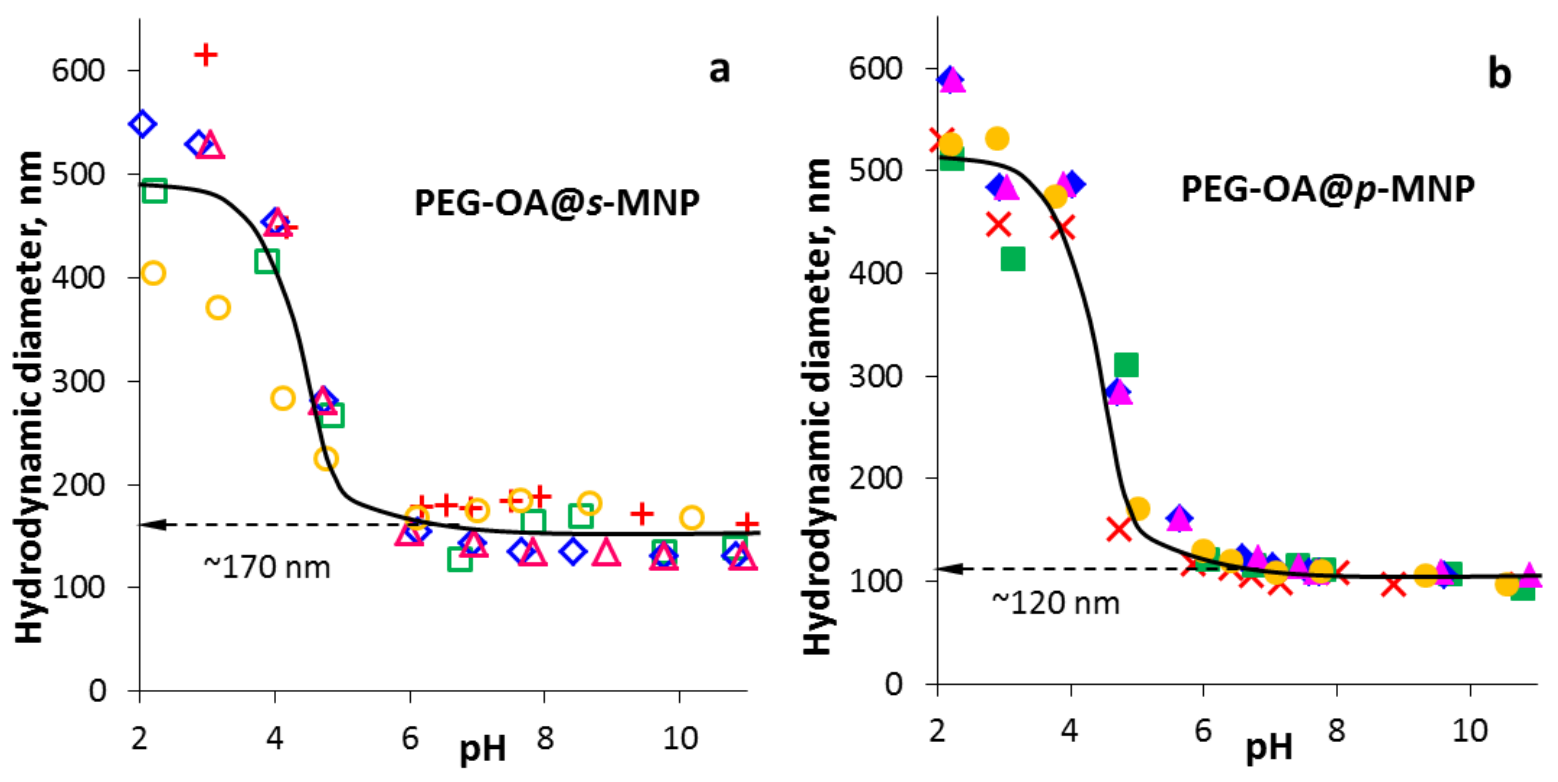

Fig. 5. The pH-dependent hydrodynamic diameter (Z-Ave values) of (a) PEG-OA@ $s$-MNP and (b) PEGOA@p-MNP samples in the presence of $10 \mathrm{mM} \mathrm{NaCl}$. The symbols indicate PEG loadings of 0 (red crosses $\mathrm{x}$ for the $p$-MNP and + for the $s$-MNP samples), 5 (green square), 15 (blue diamond), 50 (magenta triangle) and 120 (orange circle) mmol EO/g; open symbols: PEG-OA@ @-MNPs, full symbols: PEG-OA @ p-MNPs. For aggregated samples $(\mathrm{pH}<6)$, the measured points represent a given kinetic stage of the coagulating systems. (Lines are drawn as a guide for the eye.)

Table 1. Mean values of hydrodynamic diameter (Z-Ave), polydispersity index (PDI) and electrokinetic potential (Zeta) values of different $p$-MNPs at $\mathrm{pH} 6.5$ and $\mathrm{I}=10 \mathrm{mM}$. The amount of PEG of different molecular weights $(1000,4000$ and $20000 \mathrm{Da})$ is $5 \mathrm{mmol} \mathrm{EO} / \mathrm{g}$.

\begin{tabular}{lccc}
\hline Sample name & Z-Ave $(\mathbf{n m})$ & PDI & Zeta $(\mathbf{m V})$ \\
\hline$p$-MNP & 98 & 0.147 & $\mathbf{1 4}$ \\
\hline OA@ $p$-MNP & 121 & 0.169 & $\mathbf{- 5 6}$ \\
PEG1000-OA@p-MNP & 131 & 0.166 & $\mathbf{- 4 1}$ \\
PEG4000-OA@p-MNP & 124 & 0.128 & $\mathbf{- 3 5}$ \\
PEG20000-OA@ $p$-MNP & 121 & 0.141 & $\mathbf{- 3 2}$
\end{tabular}

It is seen in Table 1 that while the measured Z-Ave values are practically independent of PEG addition at $\mathrm{pH}>6$, the absolute values of zeta potentials decrease with increasing amount of PEG (see also in Fig. 4). The precision of the hydrodynamic diameters (Z-Ave) was about $\pm 20 \mathrm{~nm}$ as calculated by the size-distribution analysis software of the NanoZS instrument, so the mean values of size data given in Table 1 should be considered with caution. The larger polymers form thicker adsorbed layers as compared to the shorter chain molecules in general, because they can develop longer loops and tails on the particle surface. However, the probable conformation of the fully extended hydrated PEG chains in aqueous medium is helical (stabilized by H-bonded water molecules) [46] and its relative rigidity restricts loop and tail formation. In this regard, it is not surprising that the adsorbed layer thickness of both smaller and larger PEG molecules is similar (within the margin of error) at the same PEG loadings as seen in Table 1 for $5 \mathrm{mmol} \mathrm{EO/g} \mathrm{MNP.} \mathrm{Although} \mathrm{the}$ electrostatic repulsion is diminished gradually by the increasing thickness of PEG coating, steric repulsion between the highly solvated PEG-coated particles takes over its role in colloidal stabilization. As the weight of the electrostatic contribution in the originally electrosteric stabilization mechanism decreases and that of the steric contribution increases, the stability remains practically unchanged. 


\subsection{Salt tolerance of the core-shell MNPs}

The kinetics of coagulation of the naked, OA and PEG-OA coated $p$-MNPs in $\mathrm{NaCl}$ medium was measured to determine the critical coagulation concentrations at near-physiological $\mathrm{pH} \sim 6.5$. The evolution of the hydrodynamic diameter of the particles was followed in time by dynamic light scattering experiments, an example of which is presented in Fig. $6 \mathrm{a}$ and the corresponding evaluation for obtaining CCC in Fig. 6b. The resulting CCC values for the $p$-MNP samples (uncoated, OA and PEG1000-, PEG4000- and PEG20000-OA coated at 5, 15, 50 and $120 \mathrm{mmol} \mathrm{EO/g}$ of PEG loadings) are presented in Fig. 7.
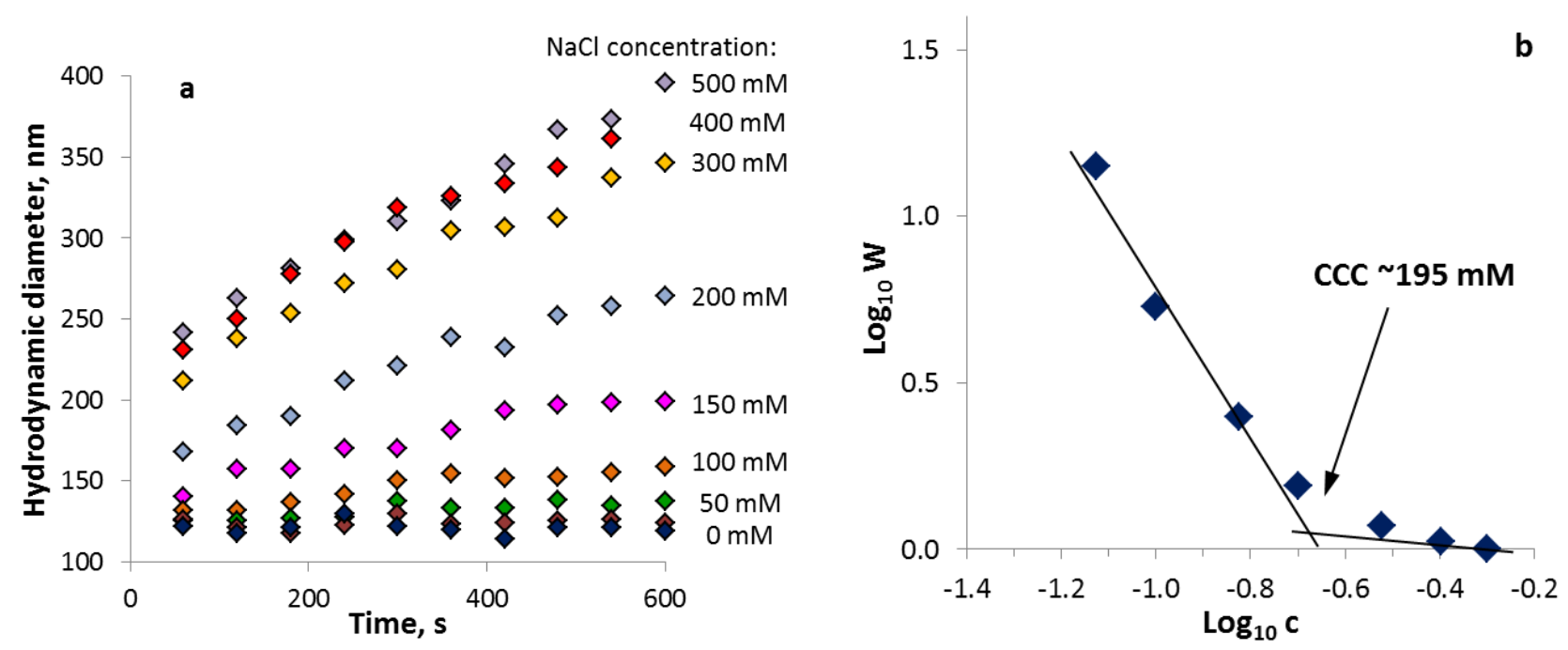

Fig 6. (a) Time-dependent increase in size of the PEG4000-OA@p-MNP sample at pH 6.5 and increasing $\mathrm{NaCl}$ concentrations ( 0 to $500 \mathrm{mM})$ at $25 \pm 0.1^{\circ} \mathrm{C}(5 \mathrm{mmol} \mathrm{EO} / \mathrm{g}$ PEG loading) and (b) the log stability vs. $\log \mathrm{NaCl}$ concentration plot to determine the critical coagulation concentration (CCC).

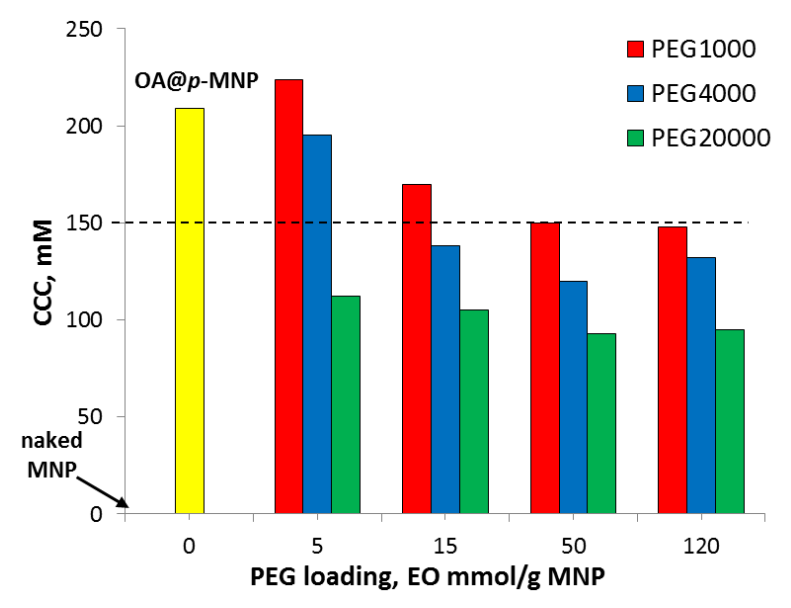

Fig 7. The effect of PEGylation on the salt tolerance of PEG-OA@p-MNP samples containing 1000, 4000 and 20000 Da molecular weight PEG at pH 6.5 and different PEG loadings $(0,5,15,50$ and $120 \mathrm{mmol}$ $\mathrm{EO} / \mathrm{g} \mathrm{MNP})$, at $25^{\circ} \mathrm{C}$. For the sake of comparison the CCC value of naked MNP ( 1 mM) is also shown. Broken line indicates physiological salt concentration.

OA-coating enhanced the salt tolerance of the uncoated MNPs from $\sim 1 \mathrm{mM}$ to about $208 \mathrm{mM}$. Adding the lowest molecular weight PEG1000 in the smallest amount $5 \mathrm{mmol} / \mathrm{g}$ resulted in somewhat higher CCC value $(225 \mathrm{mM})$. The same amount of PEG of increasingly higher molecular weights (4000 and 20000), however, reduced the CCC significantly (for the PEG20000, well below physiological salt concentration 150 
$\mathrm{mM})$. In addition, PEG1000 also decreased the salt tolerance at higher loadings 15,50 and $120 \mathrm{mmol} / \mathrm{g}$. The optimum stabilization efficiency, thus, was found for the $5 \mathrm{mmol} / \mathrm{g}$ loading of PEG1000. The decrease in the salt tolerance with both increasing PEG molecular weight and PEG loading can be explained by the effect of its highly hydrophilic character. As more ethylene oxide groups are introduced into the dispersions to build increasingly thicker coating shells in order to enhance colloidal stability, the activity of water in the aqueous medium decreases, expressing an increase in effective salt concentration.

\subsection{MRI contrast enhancement}

The potential biomedical applicability of the PEGylated core-shell nanoparticles was tested in MRI measurements at $1.5 \mathrm{~T}$. An example of determination of $T_{l}$ relaxation times from the absolute values of signal intensities vs. inversion time functions obtained for increasing iron concentrations is presented in Fig. 8a for the PEG20000-OA@s-MNP at PEG loading of $120 \mathrm{mmol} / \mathrm{g}$. The curves show the decrease in the period of the signal from the initial magnetization level. The increase in the iron concentration of the magnetic fluids resulted in longitudinal relaxation shortening, i.e., in the acceleration of the relaxation of water molecules. Similar trend was observed for all the other studied MNP samples.

From the exponential fitting of the signal intensity vs. inversion time functions the $T_{1}$ relaxation times, the relaxation rates $\left(1 / T_{1}\right)$ and the spin-lattice relaxivities $\left(r_{l}\right)$ were determined. The $1 / T_{1}$ values showed linear dependence on the iron concentration and the slope gives the $r_{l}$ values (Fig. 8b). The spin-lattice relaxivities are characteristic only to the material's quality.
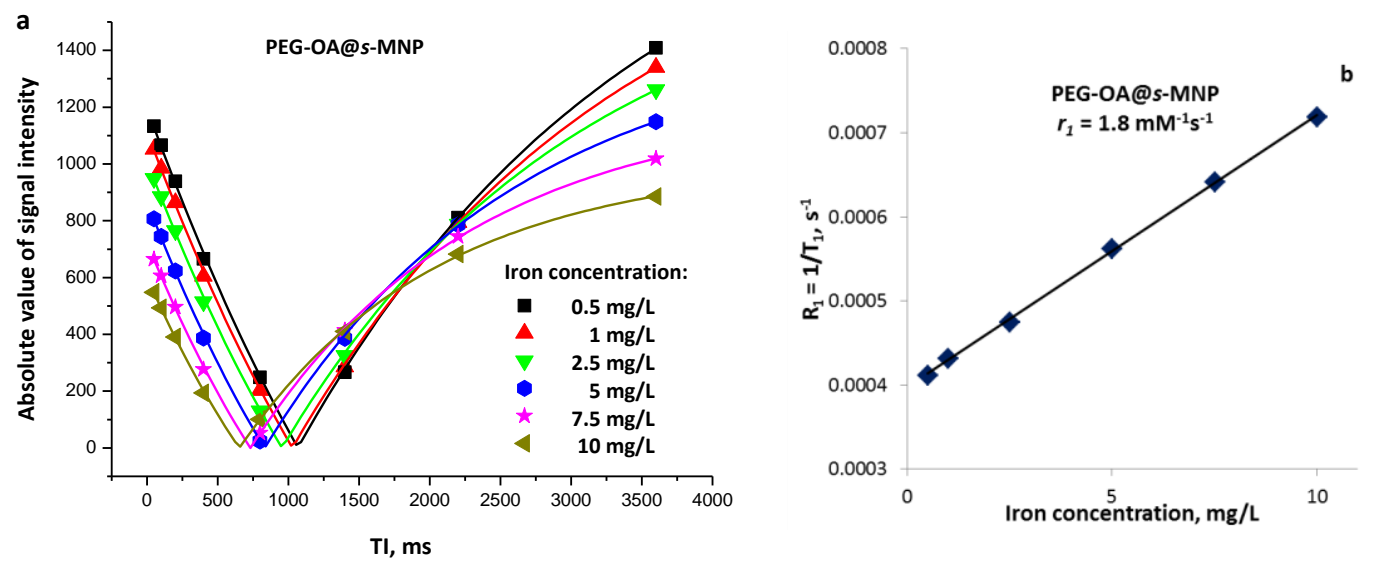

Fig. 8. (a) Longitudinal $\left(T_{1}\right)$ relaxation curves of PEG-OA@ $s$-MNPs (PEG20000 at $120 \mathrm{mmol}$ EO/g loading) in phantom water at various iron concentrations at $\mathrm{pH} 6.5$ and $\mathrm{I}=10 \mathrm{mM}$. The data are the mean signal intensities within ROI as measured at $1.5 \mathrm{~T}$ and $20^{\circ} \mathrm{C}$. The symbols correspond to experimental data and the lines are exponential fits (as explained in section 2.7). (b) Longitudinal relaxation rates $\left(R_{l}=1 / T_{1}\right)$ as a function of iron concentration of the same sample and the resulting $r_{l}$ relaxivity value.

An example of the recorded signal intensities in the function of the echo time (TE, ms) is shown in Fig. 9a measured for the PEG-OA@s-MNP. After turning off the excitation, the transversal magnetization decays exponentially to $37 \%$ of the original signal intensity. The signal decay becomes steeper with increasing iron concentration indicating spin-spin $\left(T_{2}\right)$ relaxation shortening [2-9]. The $r_{2}$ relaxivities were calculated as the slope of the function of the transverse relaxation rate $\left(1 / T_{2}\right)$ vs. iron concentration as presented in Fig. $9 \mathrm{~b}$. 

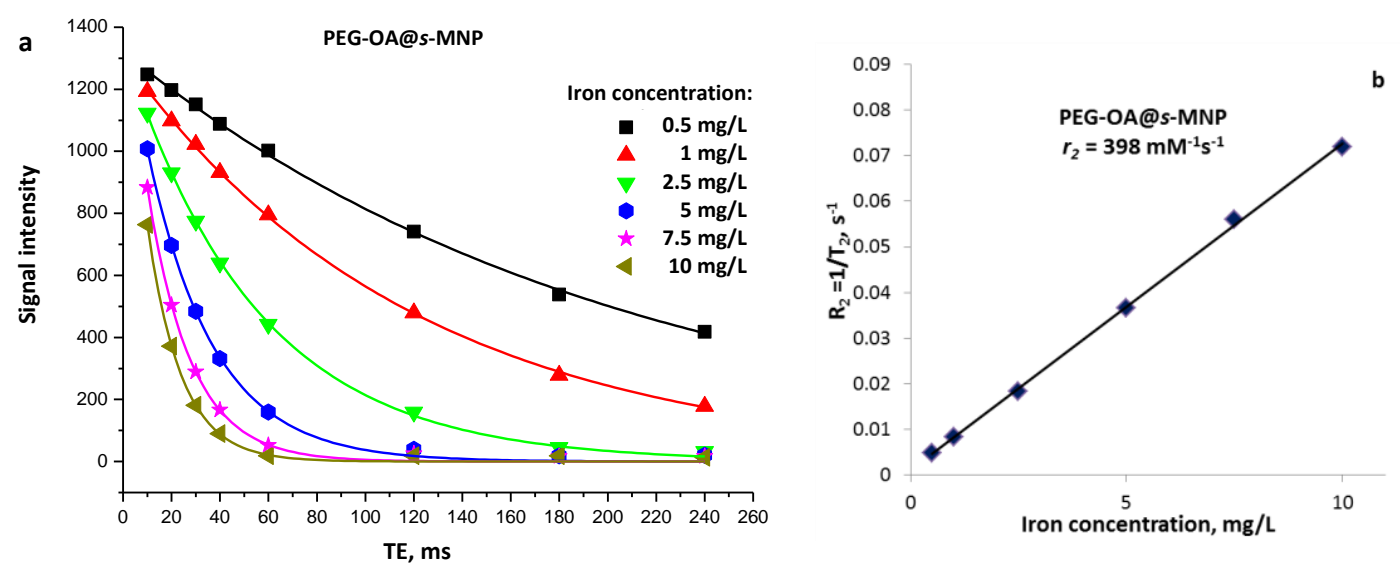

Fig. 9. (a) Transverse $\left(T_{2}\right)$ relaxation curves of PEG-OA@ $s$-MNPs (PEG20000 at $120 \mathrm{mmol}$ EO/g loading) in phantom water at various iron concentrations at $\mathrm{pH} \sim 6.5$ and $\mathrm{I}=10 \mathrm{mM}$. The data are the mean signal intensities within ROI as measured at $1.5 \mathrm{~T}$ and $20^{\circ} \mathrm{C}$. The symbols correspond to experimental data and the lines are exponential fits (as explained in section 2.7). (b) Transverse relaxation rate $\left(R_{2}=1 / T_{2}\right)$ of the same sample as a function of iron concentration and the resulting $r_{2}$ relaxivity value.

The goodness of fit was good enough both for the $1 / T_{1}$ and $1 / T_{2}$ functions vs. iron concentration (Rsquared varied between 0.98 and 0.99); thus, the $r_{1}$ and $r_{2}$ values are reliable of our magnetite nanoparticles. The $r_{1}$ and $r_{2}$ values obtained for the OA@ $s$-MNP, PEG-OA@ $s$-MNP, OA@p-MNP and PEG-OA@p-MNP samples are summarized in Table 2. For comparison, data for the commercially available Resovist and GdDTPA are also included.

Table 2. The $r_{1}$ and $r_{2}$ relaxivities of magnetic fluids with different surface coatings determined at $1.5 \mathrm{~T}$ and $20{ }^{\circ} \mathrm{C}$

\begin{tabular}{lccc}
\hline Sample name (hydrodynamic size, $\mathrm{nm})$ & $\boldsymbol{r}_{\mathbf{1}}\left(\mathbf{m M}^{-\mathbf{1}} \mathbf{s}^{\mathbf{- 1}}\right)$ & $\boldsymbol{r}_{\mathbf{2}}\left(\mathbf{m M}^{-\mathbf{1}} \mathbf{s}^{\mathbf{- 1}}\right)$ & $\boldsymbol{r}_{\mathbf{2}} / \boldsymbol{r}_{\boldsymbol{1}}$ \\
\hline OA@s-MNP (168) & 2.3 & 437 & 190.0 \\
PEG-OA@ -MNP (173) & 1.8 & 398 & 221.1 \\
\hline OA@p-MNP (121) & 2.1 & 246 & 117.6 \\
PEG-OA@p-MNP (121) & 1.1 & 230 & 209.1 \\
\hline Resovist (carboxydextran coated SPIO) [17] & 14.4 & 306 & 21.2 \\
Magnevist (Gd-DTPA) [17] & 4.7 & 5.3 & 1.1
\end{tabular}

As Fig. 8b and the $r_{l}$ values show the PEG-OA coated nanoparticles exhibit lower relaxation rate compared to the OA covered MNPs. In addition to the hydrophilicity of the particle's environment, the distance of the aqueous medium from the magnetic core influences greatly the relaxivities, since the detection of the relaxation processes is based on the protons of water molecules. One of the original purposes of the PEGylation was to enrich the particle's surroundings in water molecules via increasing the hydrophilicity of the nanomagnets. Considering that the formation of a PEG top shell leads to the extension of the distance of water molecules from the magnetic centre resulting longer relaxation times, it is not surprising that PEG adsorption slightly decreased the $r_{l}$ values for both the $s$-MNP and $p$-MNP particles (2.3 to 1.8 and 2.1 to 1.1, respectively). 

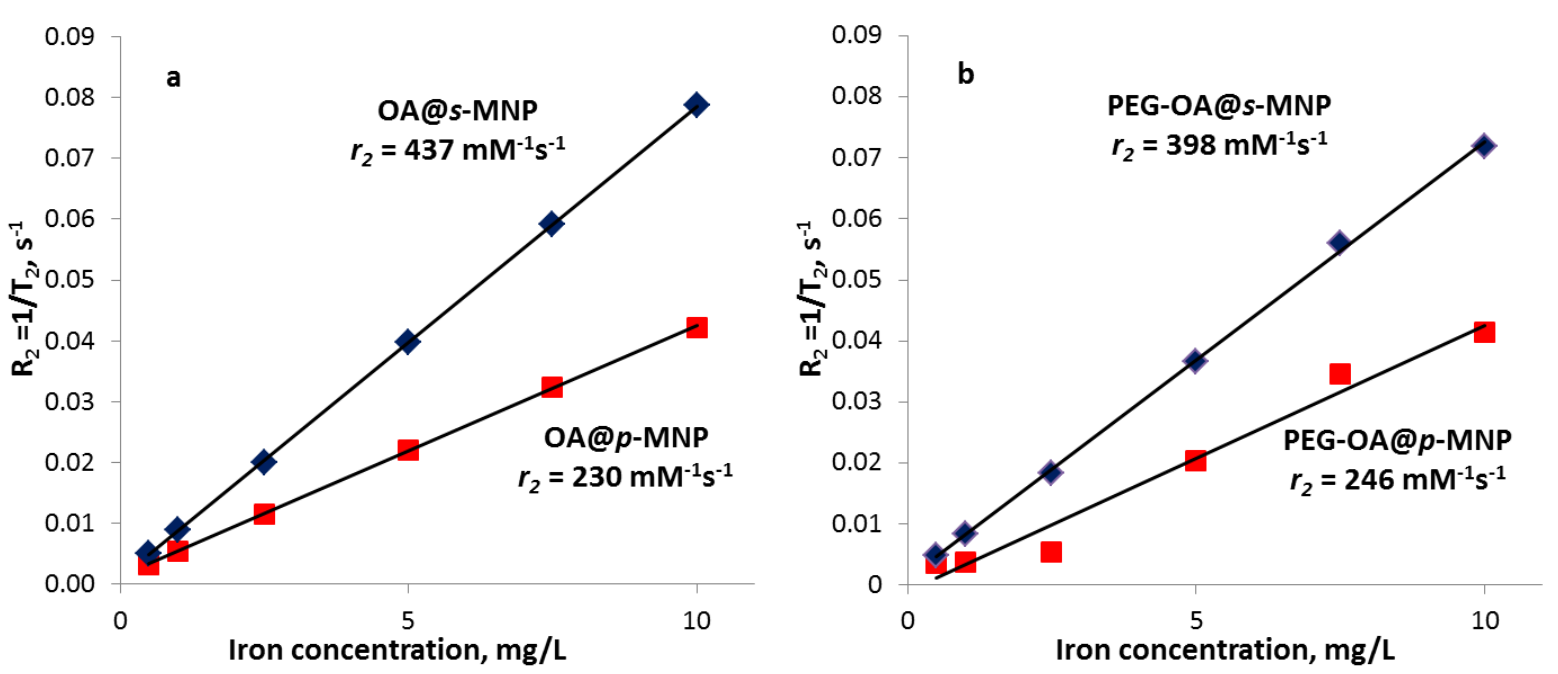

Fig. 10. Transverse relaxation rates $\left(1 / T_{2}\right)$ of (a) the surfacted $s$-MNPs and $p$-MNPs and (b) PEG-surfacted $s$-MNPs and $p$-MNPs with PEG20000 top shell at $120 \mathrm{mmol} \mathrm{EO/g}$ loading as a function of iron concentration measured at $\mathrm{pH} \sim 6.5$ and $10 \mathrm{mM} \mathrm{NaCl}$.

The $r_{2}$ relaxivity values obtained for OA@s-MNPs and PEG-OA@s-MNPs are high, exceeding considerably the majority of data published for other magnetic fluids $[17,26,47,48]$ as indicated in Table 2 for the case of Resovist and Gd-DTPA. It is probably due to the effect of inhomogeneities induced by the nanoparticles in the surrounding magnetic field influencing the relaxation rate of water's protons. Analogously with the longitudinal relaxation results, the PEG-OA coated nanomagnets exhibit slightly lower $r_{2}$ values than the OA covered MNPs. The possible explanation for this can be that the distance of water molecules from the magnetic core increases with the increasing thickness of the adsorbed PEG layer as explained above.

The remarkable difference between the $r_{2}$ relaxivities of the core-shell nanostructures based on assynthesized and purified MNPs (Table 2 and Fig. 10) can be explained by the difference in their average sizes measured by dynamic light scattering. Since $r_{2}$ strongly correlates with the hydrodynamic diameter $[26,47,48]$, higher relaxivities and greater $r_{2} / r_{1}$ ratios (Table 2) can be recorded for larger particles. The possible explanation for bigger sizes might be the slight aggregation of the sample, i.e. the clustering of particles after the coating by OA and PEG. In addition, according to Huang et al. [47], magnetic nanoparticles designed to MRI applications are in general in the "motional averaging regime" (MAR), where the larger nanomagnets possess larger magnetization resulting in higher $r_{2}$ relaxivities and $r_{2} / r_{1}$ ratios. Shao et al. [48] found a high $r_{2}$ value similar to our results $\left(420 \mathrm{mM}^{-1} \mathrm{~s}^{-1}\right)$ for large magnetic core $\mathrm{MnFe}_{2} \mathrm{O}_{4}$, which was explained by the linear quadratic relationship between the magnetization of the particles and the measurable transverse relaxivity. The $r_{2}$ values of the as-synthesized MNP based core-shell nanoparticles are almost twice of that of the pure MNP based ones suggesting considerably larger particle size, which is supported also by the Z-Ave values obtained in dynamic light scattering experiments. As discussed in section 3.3, the final particle size is likely due to cluster formation from both the $s$-MNP and $p$-MNP particles during the OA adsorption. The degree of clustering is greater in the case of as-synthesized MNPs probably because of the high salt content of the synthesis medium, in which the OA adsorption proceeds.

\subsection{Biocompatibility tests}

Blood sedimentation experiments were performed to test the biocompatibility of PEGylated nanomagnets through their erythrocyte cytotoxicity. The tubes containing the blood of Donor \#3 with PEGOA@p-MNPs, as an example, are shown in Fig. 11. The molecular weight of PEG was 1000, 4000 and 20000 $\mathrm{Da}$ in the tubes. As it can be seen, the level of the RBC's sediments is practically the same in all the MNPcontaining samples and the reference blood sample. 
The ESR measurements also support this in that all the obtained ESR values agreed within the margin of error $( \pm 3 \mathrm{~mm} / \mathrm{h})$. Thus, addition of the PEGylated nanoparticles did not affect the rate of erythrocyte sedimentation meaning that the colloidal state of the human blood remained unchanged. We observed also a dark brown colour appearing in the supernatant (blood plasma) probably due to the presence of the MNPs in it $[10,11]$.

Blood smear experiments were performed to study the haemolytic potential of the PEG-surfacted coreshell MNPs, in which the magnetic fluids were mixed human blood at 1:5 volumetric ratios. The pictures of the plasma collected by centrifugation of these mixtures are shown in Fig. 12.

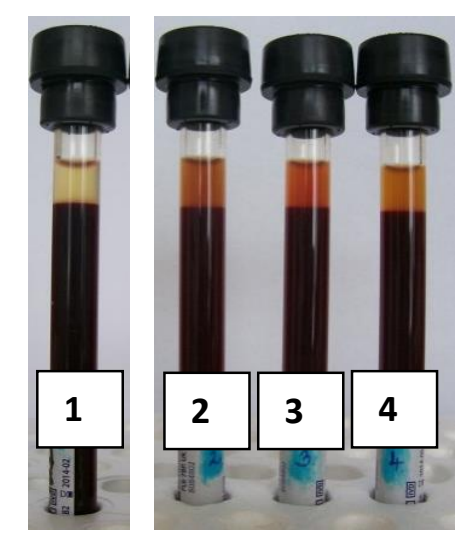

Fig. 11. Representative blood sedimentation experiment: (1) is the control sample of Donor \#3 and (2), (3) and (4) are the samples after addition of PEG-OA@p-MNPs (the loadings are $5 \mathrm{mmol} \mathrm{EO/g}$ of PEG of 1000, 4000 and $20000 \mathrm{Da}$, respectively) at final concentration of $0.16 \mathrm{~g} / \mathrm{L} \mathrm{MNP}$.

No change in the shape of the red blood cells (RBCs) can be observed even at high iron oxide content as seen in the microscopic picture taken after addition of the PEGylated nanomagnets indicating the absence of thrombocyte aggregation. The colour of the plasma changed from yellow to brown because of the presence of magnetic nanoparticles in the supernatant. All of the OA@MNP and PEG-OA@MNP samples contacted with all of the tested blood samples showed the complete absence of blood-coagulating or haemolytic potential in harmony with experiences published by others $[5,10,11,20,23,26,28]$.

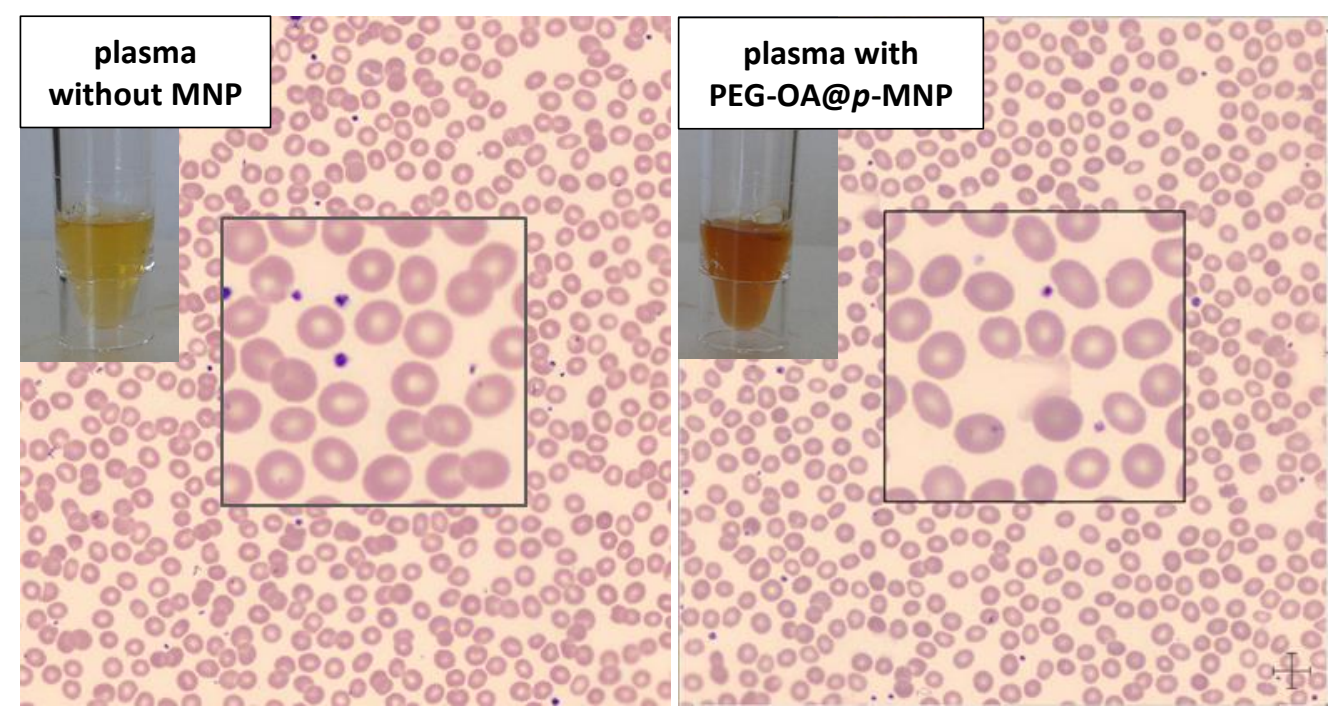

Fig. 12. Smears of human blood (a) before and (b) after the addition the PEG-OA@p-MNP (PEG20000 loading of $5 \mathrm{mmol} \mathrm{EO} / \mathrm{g}$ ). The insets show the blood plasma with and without the PEGylated nanomagnets. 


\section{Conclusions}

Preparation of surfacted MNPs is one of the oldest methods or synthesis of magnetic fluids. The most widespread oleic acid coated MNPs are hydrophobic, but one can easily convert them into hydrophilic counterpart by adsorption of a second oleic acid layer even in the original pot of synthesis. The double layer coated MNPs dispersed in water at biorelevant pHs (e.g. 7.4 in blood) carry strongly interacting carboxylate groups of the outer oleate anion (OA) layer. However, because of the long history of this type of synthesis, versatile strategies circulate in literature without much intention to compare their actual success in terms of the overall quality of products. In this work, our aim was to provide quantitative comparison of the colloidal stability, salt tolerance, MRI contrasting ability and biocompatibility of PEG-surfacted MNPs produced in two principally different ways: either creating oleate double layer in situ in the synthesis mixture of MNPs or by coating highly purified MNPs by designed amount of oleate. The question whether or not the synthesis conditions influence fundamentally the properties of the MNP products has been answered in this paper.

The results suggest first that significantly different products can be obtained by the two synthesis methods, albeit not in all aspects. The main finding is that the adsorption of the first oleic acid layer proceeds via different mechanisms; namely, surface complexation takes place in the case of as-synthesized MNPs, while it is accomplished by H-bonding too, when purified MNPs react with oleate. It is noteworthy that this seemingly small difference affects the hydrodynamic diameter of the oleate anion coated MNPs (OA@MNPs), which can be ascribed to the different cluster formation tendencies of the primary OA@MNPs. The low pH and ionic strength of the purified MNPs' medium are beneficial to H-bond formation and appear to counteract cluster formation; while the high salt concentration in the synthesis mixture of as-synthesized MNPs promotes clustering. The significant difference in the degrees of clustering was manifested in the $r_{2}$ relaxation values, the as-synthesized samples showing twice as strong contrasting ability than the purified ones. The second interesting result of the present study is related to the stabilization mechanism of the PEG layers adsorbed on electrically charged nanoparticle surfaces. The changes in electrokinetic potential and hydrodynamic diameter of OA@MNPs during PEG adsorption were measured and we found that although electrokinetic potential decreases gradually due to the emerging PEG layer, the hydrodynamic size of PEGylated particles does not change appreciably. It seems that MNPs aggregate since the electrostatic repulsion is weakened; meanwhile they remain colloidally stable likely because of increasing steric repulsion. We can conclude that the original electrosteric stabilization by the OA double layer has a greater contribution from-electrostatic component, but the forming PEG top layer enhances the steric contribution.

In general, the PEG-OA @ MNPs prepared according to the studied methods were well stabilized at the conditions $\mathrm{pH} \sim 6.5$ and $\mathrm{I}=10 \mathrm{mM}$. The high measured $\mathrm{CCC}$ values ensure that their salt tolerance is sufficiently high to resist aggregation in physiological medium. The samples were all biocompatible that is a final requirement for their possible biomedical applicability.

\section{Acknowledgements}

This work was supported by OTKA (NK 84014) and TÁMOP-4.2.2.A-11/1/KONV-2012-0047 grants. This material was presented at the $27^{\text {th }}$ ECIS Conference and the first author of this paper was awarded one of the ECIS2013 Elsevier Poster Prizes. 


\section{References}

[1] R.M. Cornell, U. Schwertmann, The Iron Oxides, VCH,Weimheim, 1996.

[2] Q.A. Pankhurst, N.K.T. Than, S.K. Jones, J. Dobson, Progress in applications of magnetic nanoparticles in biomedicine, J. Phys. D: Appl. Phys. 42 (2009) 224001 (15pp).

[3] O. Veiseh, J.W. Gunn, M. Zhang, Design and fabrication of magnetic nanoparticles for targeted drug delivery and imaging, Adv. Drug. Deliver Rev. 62 (2010) 284-304.

[4] J.K. Oh, J.M. Park, Iron oxide-based superparamagnetic polymeric nanomaterials: Design, preparation, and biomedical application, Progr. Polym. Sci. 36 (2011) 168-189.

[5] E.J. Rosen, L. Chan, D.-B. Shieh, F.X. Gu, Iron oxide nanoparticles for targeted cancer imaging and diagnostics, Nanomedicine 8 (2012) 275-290.

[6] C. Corot, D. Warlin, Superparamagnetic iron oxide nanoparticles for MRI: contrast media pharmaceutical company R\&D perspective. Wiley Interdiscip Rev Nanomed Nanobiotechnology, 2013.

[7] B. Mishra, B.B. Patel, S. Tiwari, Colloidal nanocarriers: a review on formulation technology, types and applications toward targeted drug delivery, Nanomed-Nanotechnol 6 (2010) 9-24.

[8] M. Mahmoudi, S. Sant, B. Wang, S. Laurent, T. Sen, Superparamagnetic iron oxide nanoparticles (SPIONs): development, surface modification and applications in chemotherapy, Adv. Drug Delivery Rev. 63 (2011) 24-46.

[9] S. Mura, P. Couvreur, Nanotheranostics for personalized medicine, Adv. Drug Delivery Rev. 64 (2012) 1394-1416.

[10] I.Y. Tóth, E. Illés, R.A. Bauer, D. Nesztor, M. Szekeres, I. Zupkó, E. Tombácz, Designed polyelectrolyte shell on magnetite nanocore for dilution-resistant biocompatible magnetic fluids, Langmuir 28 (2012)16638-16646.

[11] M. Szekeres, I.Y. Tóth, E. Illés, A. Hajdú, I. Zupkó, K. Farkas, G. Oszlánczi, L. Tiszlavicz, E. Tombácz, Int. J. Mol. Sci. 14 (2013) 14550-14574.

[12] D. Bica, L. Vékás, M.V. Avdeev, O. Marinica, V. Socoliuc, M. Balasoiu, V.M. Garamus, Sterically stabilized water based magnetic fluids: Synthesis, structure and properties. J. Magn. Magn. Mater. 311 (2007) 17-21.

[13] A. Jozefczak, A. Skumiel, Ultrasonic investigation of magnetic nanoparticles suspension with PEG biocompatible coating, J. Magn. Magn. Mater. 323 (2011) 1509-1516.

[14] E. Tombácz, D. Bica, A. Hajdú, E. Illés, A. Majzik, L.Vékás, Surfactant double layer stabilized magnetic nanofluids for biomedical application, J. Phys. Cond. Matter 20 (2008) Article ID 20410 (6 pages)

[15] A. Hajdú, E. Tombácz, E. Illés, D. Bica, L. Vékás, Magnetite nanoparticles stabilized under physiological conditions for biomedical application, Prog. Colloid Polym. Sci. 135 (2008) 29-37.

[16] M.V. Avdeev, A.V. Feoktystov, P. Kopcansky, G. Lancz, V.M. Garamus, R. Willumeit, M. Timko, M. Koneracka, V. Zavisova, N. Tomasovicova, A. Jurikova, K. Csach, L.A. Bulavin, Structure of waterbased ferrofluids with sodium oleate and polyethylene glycol stabilization by small-angle neutron scattering: contrast-variation experiments, J. Appl. Cryst. 43 (2010) 959-969.

[17] A. Jedlovszky-Hajdú, E. Tombácz, I. Bányai, M. Babos, A. Palkó, Carboxylated magnetic nanoparticles as MRI contrast agents: Relaxation measurements at different field strengths, J. Magn. Magn. Mater. 324 (2012) 3173-3180.

[18] V. Zavisova, M. Koneracka, M. Muckova, J. Lazova, A. Jurıkova, G. Lancz, N. Tomasovicova, M. Timko, J. Kovac, I. Vavra, M. Fabian, A.V. Feoktystov, V.M. Garamus, M.V. Avdeev, P. Kopcansky, Magnetic fluid poly(ethylene glycol) with moderate anticancer activity, J. Magn. Magn. Mater. 323 (2011) 1408-1412.

[19] A. Jurikova, K. Csach, J. Miskuf, M. Koneracka, V. Zavisova, M. Kubovcikova, P. Kopcansky, M. Muckova, Thermal Properties of Magnetic Nanoparticles Modified With Polyethylene Glycol, Magnetics, IEEE Transactions 49 (2013) 236-239.

[20] M. Mahdavi, M.B. Ahmad, M.J. Haron, F. Namvar, B. Nadi, M.Z.A. Rahman, J. Amin, Synthesis, Surface Modification and Characterisation of Biocompatible Magnetic Iron Oxide Nanoparticles for Biomedical Applications, Molecules 18 (2013) 7533-7548. 
[21] E. Tombácz, I.Y. Tóth, D. Nesztor, E. Illés, A. Hajdú, M. Szekeres, L. Vékás, Adsorption of organic acids on magnetite nanoparticles, pH-dependent colloidal stability and salt tolerance, Coll. Surf. A 435 (2013) 91-96.

[22] L.M. Armijo, Y.I. Brandt, D. Mathew, S. Yadav, S. Maestas, A.C. Rivera, N.C. Cook, N.J. Withers, G.A. Smolyakov, N.L. Adolphi, T.C. Monson, D.L. Huber, H.D.C. Smyth, M. Osiński, Iron Oxide Nanocrystals for Magnetic Hyperthermia Applications, Nanomaterials 2 (2012) 134-146.

[23] L. Sun, C. Huang, T. Gong, S. Zhou, A biocompatible approach to surface modification: Biodegradable polymer functionalized super-paramagnetic iron oxide nanoparticles, Mat. Sci. Eng. C 30 (2010) 583589.

[24] S. García-Jimeno, J. Estelrich, Ferrofluid based on polyethylene glycol-coated iron oxide nanoparticles: Characterization and properties, Coll. Surf. A 420 (2013) 74-81.

[25]B. Thong-On, B. Rutnakornpituk, U. Wichai, M. Rutnakornpituk, Magnetite nanoparticle coated with amphiphilic bilayer surfactant of polysiloxane and poly(poly(ethylene glycol) methacrylate), J Nanopart. Res. 14:953 (2012) (12 pages)

[26] U.I. Tromsdorf, O.T. Bruns, S.C. Salmen, U. Beisiegel, H. Weller, A Highly Effective, Nontoxic T1 MR Contrast Agent Based on Ultrasmall PEGylated Iron Oxide Nanoparticles, Nano Letters 9 (2009) 44344440.

[27] X. Cao, B. Zhang, F. Zhao, L. Feng, Synthesis and Properties of MPEG-Coated Superparamagnetic Magnetite Nanoparticles, J. Nanomaterials 2012 (2012), Article ID 607296 (6 pages).

[28] A. Masoudi, H.R.M. Hosseini, M.A. Shokrgozar, R. Ahmadi, M.A. Oghabian, The effect of poly(ethylene glycol) coating on colloidal stability of superparamagnetic iron oxide nanoparticles as potential MRI contrast agent, Int. J. Pharm. 433 (2012) 129- 141.

[29] V.I. Shubayev, T.R. Pisanic, S. Jin, Magnetic nanoparticles for theragnostics, Adv. Drug Delivery Rev. 61 (2009) 467-477.

[30]D.C. Ferreira Soares, M.C. de Oliveira, R.G. dos Santos, M.S. Andrade, J.M.C. Vilela, V.N. Cardoso, G.A. Ramaldes, Liposomes radiolabeled with 159Gd-DTPA-BMA: Preparation, physicochemical characterization, release profile and in vitro cytotoxic evaluation. Eur. J. Pharm. Sci. 42 (2011) 462-469.

[31] M.C. Heinrich, M.K. Kuhlmann, S. Kohlbacher, M. Scheer, A. Grgic, M:B. Heckmann, M. Uder, Cytotoxicity of iodinated and gadolinium-based contrast agents in renal tubular cells at angiographic concentrations: In vitro study, Radiology 242 (2007) 425-434.

[32] M.E. Bartolini, J. Pekar, D.R. Chettle, F. McNeill, A. Scott, J. Sykes, F.S. Prato, G.R. Moran, An investigation of the toxicity of gadolinium based MRI contrast agents using neutron activation analysis. Magn. Reson. Imaging 21 (2003) 541-544.

[33] M.L. Etheridge, S.A. Campbell, A.G. Erdman, C.L. Haynes, S.M. Wolf, J. McCullough, J. Nanomedicine: Nanotechnology, Biology and Medicine 9 (2013) 1-14.

[34] L. Vékás, D. Bica, O. Marinica, Magnetic nanofluids stabilized with various chain length surfactants, Rom. Repts. Phys. 58 (2006) 217-228.

[35] E. Illés, E. Tombácz, The effect of humic acid adsorption on pH-dependent surface charging and aggregation of magnetite nanoparticles. J. Colloid Interface Sci. 295 (2006) 115-123.

[36]D.J. Shaw, Introduction to Colloid and Surface Chemistry, Butterworths, London, 1966.

[37] M. Elimelech, J. Gregory, X. Jia, R.A. Williams, Particle Deposition and Aggregation: Measurement, Modeling and Simulation, Butterworths, Oxford, 1995.

[38] R. Kretzschmar, H. Holthoff, H. Sticher, Influence of $\mathrm{pH}$ and humic acid on coagulation kinetics of kaolinite: a dynamic light scattering study, J. Colloid Interface Sci. 202 (1998) 95-103.

[39] C. Henoumont, S. Laurent, L.Vander Elst, How to perform accurate and reliable measurements of longitudinal and transverse relaxation times of MRI contrast media in aqueous solutions, Contrast Media and Molecular Imaging 4 (2009) 312-321.

[40] A. Roch, Y. Gossuin, R.N. Muller, P. Gillis, Superparamagnetic colloid suspensions: water magnetic relaxation and clustering, J. Magn. Magn. Mater. 293 (2005) 532-539.

[41] R. Baigorri, J.M Garcia-Mina, G. Gonzalez-Gaitano, Supramolecular association induced by Fe(III) in low molecular weight sodium polyacrylate, Coll. Surf. A 292 (2007) 212-216.

[42] S. Liufu, H. Xiao, Y. Li, Adsorption of poly(acrylic acid) onto the surface of titanium dioxide and the colloidal stability of aqueous suspension, J. Coll. Interface Sci. 281 (2005) 155-163. 
[43] R. Bywalez, H. Karacuban, H. Nienhaus, C. Schulz, H. Wiggers, Stabilization of mid-sized silicon nanoparticles by functionalization with acrylic acid, Nanoscale Research Letters 7:76 (2012)(7 pages).

[44] J.J. Najera, Phase transition behaviour of sodium oleate aerosol particles, Atmospheric Environment 41 (2007) 1041-1052.

[45] P.J. Larkin, Infrared and Raman Spectroscopy; Principles and Spectral Interpretation. London, Elsevier, 2011.

[46] Encyclopedia of Surface and Colloid Science, Vol. 7. (Ed. P. Somasundaran), CRC Press, Boca Raton, USA, 2006. p. 4644.

[47] J. Huang, X. Zhong, L. Wang, L. Yang, H. Mao, Improving the Magnetic Resonance Imaging Contrast and Detection Methods with Engineered Magnetic Nanoparticles, Theranostics 2 (2012) 86-102.

[48] H. Shao, C. Min, D. Issadore, M. Liong, T.-J. Yoon, R. Weissleder, H. Lee, Magnetic Nanoparticles and microNMR for Diagnostic Applications, Theranostics 2 (2012) 55-65. 